09.1

\title{
Излучение дробного осциллятора
}

\author{
() С.Ш. Рехвиашвили ${ }^{1}$, А.В. Псху ${ }^{1}$, 3.Ч. Маргушев ${ }^{2}$ \\ ${ }^{1}$ Институт прикладной математики и автоматизации КБНЦ РАН, Нальчик, Россия \\ ${ }^{2}$ Институт инорорматики и проблем регионального управления КБНЦ РАН, Нальчик, Россия \\ E-mail: rsergo@mail.ru
}

Поступило в Редакцию 20 июля 2021 г.

В окончательной редакции 29 июля 2021 г.

Принято к публикации 15 августа 2021 г.

В дипольном приближении вычислена спектральная плотность энергии излучения осциллятора, движение которого описывается уравнением с дробным интегро-дифференцированием. Модель дробного осциллятора может описывать различные типы излучения, в том числе и с неэкспоненциальным законом релаксации. Найдена форма спектральной линии излучения. Проведено сравнение полученного результата с классическим лоренцевским спектром и экспериментальными спектрами излучения монохроматического и люминофорного светодиодов. Порядок дробного интегро-дифференцирования в модели задает величину уширения спектра излучения.

Ключевые слова: дробный осциллятор, дробное интегро-дифференцирование, контур спектральной линии, дипольное излучение.

DOI: 10.21883/PJTF.2021.22.51728.18964

Математическая модель дробного осциллятора (от англ. fractional oscillator) может конструктивно применяться для описания колебательных процессов как с медленным, так и с быстрым затуханием [1-17]. Суть этой модели заключается в том, что закон эволюции механической или динамической системы задается с учетом эффекта динамической памяти: считается, что импульс системы в текущий момент времени $t$ устанавливается в результате действия каких-либо сил в предшествующие моменты времени $t^{\prime}<t$. В наших работах [11-13] было показано, что степенная функция памяти учитывает диссипацию энергии колебаний осциллятора. Уравнение движения такого осциллятора представляет собой дифференциальное уравнение дробного порядка. При этом обнаружено, что порядок дробного интегро-дифференцирования взаимосвязан с добротностью и коэффициентом затухания осциллятора. Этот вывод качественно и количественно подтвержден в работах [14-17].

Из классической электродинамики [18] и теории люминесценции [19] известно, что затухающие колебания в системе, состоящей из положительных и отрицательных зарядов, например в плазме твердого тела, являются причиной уширения спектра соответствующего дипольного излучения. Закон затухания такого излучения определяется динамикой возбужденного состояния, которая формируется в зависимости от конкретных физико-химических свойств системы и условий внешнего возбуждения. В связи с этим возникают закономерные вопросы относительно интенсивности и формы спектральной линии излучения дробного осциллятора, а также возможного применения этой модели к описанию реальных излучательных систем. Решению этих актуальных вопросов посвящена настоящая работа.
Запишем уравнение движения электрона в интегральной форме

$$
\mathbf{p}(t)=\mathbf{p}(0)+\int_{0}^{t} G\left(t-t^{\prime}\right) \mathbf{F}\left(\mathbf{r}, t^{\prime}\right) d t^{\prime},
$$

где $\mathbf{p}$ и $\mathbf{F}$ - векторы импульса и силы, $G-$ функция памяти. Выберем функцию памяти в степенном виде [20,21]:

$$
G(t)=\frac{1}{\Gamma(\alpha)}\left(\frac{\tau}{t}\right)^{1-\alpha}, \quad 0<\alpha \leqslant 1,
$$

где $\Gamma(\alpha)$ - гамма-функция Эйлера, $\tau-$ некоторая константа процесса. Подставляя (2) в (1), получаем уравнение движения дробного порядка

$$
\frac{d^{\alpha} \mathbf{p}(t)}{d t^{\alpha}}=\tau^{1-\alpha} \mathbf{F}(\mathbf{r}, t) .
$$

В (3) содержится дробная производная Капуто, которая по определению при $\alpha<1$ есть

$$
\frac{d^{n+\alpha} y(t)}{d t^{n+\alpha}}=\frac{1}{\Gamma(1-\alpha)} \int_{0}^{t} \frac{y^{(n+1)}\left(t^{\prime}\right) d t^{\prime}}{\left(t-t^{\prime}\right)^{\alpha}}, \quad n=0,1,2, \ldots
$$

Обширную информацию о дробных операторах и их применении в различных физических задачах можно отыскать в работах [22-26]. В отсутствие внешних полей из (3) следует уравнение одномерного дробного осциллятора

$$
\frac{d^{1+\alpha} x(t)}{d t^{1+\alpha}}+\omega_{0}^{1+\alpha} x(t)=0
$$

где $\omega_{0}=1 / \tau-$ частота незатухающих колебаний при $\alpha=1$. Будем предполагать, что движение электрона 

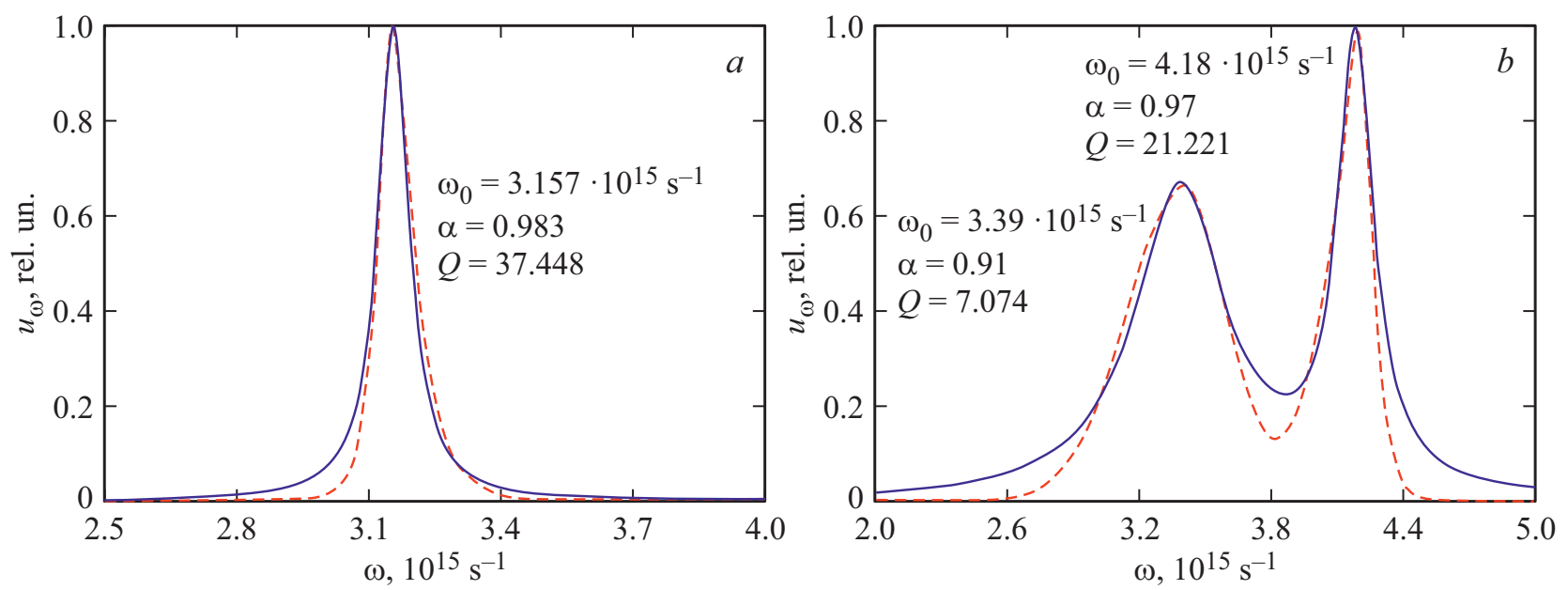

Спектры излучения монохроматического оранжевого $(a)$ и люминофорного „белого“ $(b)$ светодиодов. Штриховые кривые эксперимент, сплошные кривые - расчет по формуле (8).

и излучение начинаются в момент $t=0$. Аналогично классическому случаю $[18,19]$ примем начальные условия $x(0)=x_{0}$ и $x^{\prime}(0)=i \omega_{0} x_{0}$, где $x_{0}-$ комплексная амплитуда. Тогда решение уравнения (4) запишется в виде

$$
\begin{gathered}
x(t)=x_{0}\left\{E_{1+\alpha, 1}\left[-\left(\omega_{0} t\right)^{1+\alpha}\right]+i \omega_{0} t E_{1+\alpha, 2}\left[-\left(\omega_{0} t\right)^{1+\alpha}\right]\right\}, \\
E_{\beta, \mu}(z)=\sum_{k=0}^{\infty} \frac{z^{k}}{\Gamma(\beta k+\mu)}
\end{gathered}
$$

где $E_{\beta, \mu}(z)$ - фукция типа Миттаг-Леффлера.

Спектральная плотность энергии дипольного излучения задается формулой

$$
\begin{gathered}
u_{\omega}=\frac{q^{2}}{3 \varepsilon_{0} c^{3}}\left|\ddot{x}_{\omega}\right|^{2}, \\
\ddot{x}_{\omega}=\frac{1}{2 \pi} \int_{-\infty}^{\infty} \ddot{x}(t) \exp (i \omega t) d t,
\end{gathered}
$$

где $q$ - заряд электрона, $c$ - скорость света в вакууме, $\varepsilon_{0}$ - электрическая постоянная. В (7) подразумевается, что $x(t)=0$ при $t<0$. Из (5)-(7) получаем

$$
\begin{aligned}
& u_{\omega}=\frac{q^{2} \omega_{0}^{2(1+\alpha)}\left|x_{0}\right|^{2}}{12 \pi^{2} \varepsilon_{0} c^{3}} \\
& \times \frac{\left(\omega+\omega_{0}\right)^{2}}{\omega^{2(1+\alpha)}-2\left(\omega \omega_{0}\right)^{1+\alpha} \sin (\pi \alpha / 2)+\omega_{0}^{2(1+\alpha)}} .
\end{aligned}
$$

Для (8) имеет место условие нормировки

$$
\begin{aligned}
\int_{0}^{\infty} u_{\omega} d \omega & =\frac{q^{2} \omega_{0}^{3}\left|x_{0}\right|^{2}}{12 \pi \varepsilon_{0} c^{3}(1+\alpha)}\left[\operatorname { t a n } ( \frac { \pi \alpha } { 2 } ) \left(\csc \left(\frac{\pi}{1+\alpha}\right)\right.\right. \\
& \left.\left.-\csc \left(\frac{3 \pi}{1+\alpha}\right)\right)-2 \csc \left(\frac{2 \pi}{1+\alpha}\right)\right],
\end{aligned}
$$

в котором должно выполняться неравенство $1 / 2<\alpha<1$. Выпишем также в наших обозначениях выражение для спектра лоренцевского типа

$$
u_{\omega}=\frac{q^{2} \Omega^{4}\left|x_{0}\right|^{2}}{12 \pi^{2} \varepsilon_{0} c^{3}} \frac{1}{(\omega-\Omega)^{2}+\gamma^{2}},
$$

где $\gamma-$ коэффициент затухания, $\Omega=\sqrt{\omega_{0}^{2}-\gamma^{2}}-$ частота затухающих колебаний. В режиме слабого затухания $\omega_{0} \gg \gamma$ [19]. Для этого режима в (9) можно принять $\Omega \approx \omega_{0}$. В предельном случае при $\alpha \rightarrow 1$ и $\gamma \rightarrow 0$, т.е. в отсутствие затухания, формулы (8) и (9) совпадают.

Дальнейшая задача заключается в том, чтобы сравнить между собой спектральные линии, полученные с помощью выражений (8) и (9), а также провести сравнение с какими-либо образцовыми экспериментальными данными по излучению. Из результатов [11-13] следует, что согласование между спектральными линиями (8) и (9) должна обеспечивать приближенная формула

$$
\alpha \approx 1-\frac{2}{\pi Q}=1-\frac{4 \gamma}{\pi \omega_{0}}
$$

где $Q$ - добротность осциллятора. Численные расчеты показывают, что при соблюдении (10) удовлетворительное соответствие между (8) и (9) достигается уже при $Q>5$. При $Q \geqslant 10$ спектральные линии, рассчитанные по формулам (8) и (9), практически неразличимы. Следовательно, в режиме слабого затухания влияние динамической памяти, которая задается формулой (2), ослабевает и модель дробного осциллятора приблизительно дает экспоненциальный закон релаксации [11-13]. Математически это выражается в экспоненциальном убывании осциллирующей функции (5) в области существования ее нулей, если значение $\alpha$ близко к единице [27].

В настоящей работе была проведена экспериментальная проверка модели дробного осциллятора. В качестве 
источников излучения были выбраны SMD-светодиоды. Измерения спектров излучения светодиодов проводились с помощью спектрометра HR4000 с волоконным входом. Экспериментальные спектры излучения монохроматического оранжевого и люминофорного „белого“ светодиодов и теоретические расчеты по формуле (8) продемонстрированы на рисунке. В случае монохроматического оранжевого светодиода спектр был аппроксимирован одной спектральной линией (см. рисунок, $a$ ). Тогда как спектр люминофорного „белого“ светодиода (см. рисунок, $b$ ) аппроксимировался суммой двух спектральных линий с различными параметрами. Численные значения рассчитанных параметров для формул (8) и (10) указаны на рисунке. Можно видеть, что расчеты успешно воспроизводят основные (верхние) части спектров излучения. Для полупроводниковых светодиодов, которые можно отнести к высокодобротным излучательным системам с $Q \gg 1 / 2$, точность описания экспериментальных спектров оказывается такой же, как и в лоренцевской модели.

В заключение сформулируем основные выводы. Из сравнения расчетов с экспериментальными данными следует, что модель дробного осциллятора пригодна для описания спектральных линий дипольного излучения. Порядок дробного интегро-дифференцирования в (8) при $\alpha<1$ определяет уширение спектральной линии излучения. Главным достоинством рассмотренной модели является то, что в ней отсутствует необходимость вводить динамическую (линейно зависящую от скорости) силу трения в уравнение движения; снижение порядка дробного интегро-дифференцирования в (4) эффективно учитывает диссипацию энергии колебаний осциллятора.

\section{Конфликт интересов}

Авторы заявляют, что у них нет конфликта интересов.

\section{Список литературы}

[1] F. Mainardi, Chaos Solitons Fract., 7 (9), 1461 (1996). DOI: $10.1016 / 0960-0779(95) 00125-5$

[2] B.N. Narahari Achar, J.W. Hanneken, T. Enck, T. Clarke, Physica A, 297 (3-4), 361 (2001). DOI: $10.1016 / \mathrm{S} 0378-4371(01) 00200-\mathrm{X}$

[3] B.N. Narahari Achar, J.W. Hanneken, T. Clarke, Physica A, 309 (3-4), 275 (2002). DOI: $10.1016 / \mathrm{S} 0378-4371(02) 00609-\mathrm{X}$

[4] B.N. Narahari Achar, J.W. Hanneken, T. Clarke, Physica A, 339 (3-4), 311 (2004). DOI: 10.1016/j.physa.2004.03.030

[5] A.A. Stanislavsky, Phys. Rev. E, 70 (5), 051103 (2004). DOI: 10.1103/PhysRevE.70.051103

[6] M. Li, S.C. Lim, Sh. Chen, Math. Probl. Eng., 2011, 657839 (2011). DOI: $10.1155 / 2011 / 657839$

[7] V.E. Tarasov, Cent. Eur. J. Phys., 10 (2), 382 (2012). DOI: $10.2478 / \mathrm{s} 11534-012-0008-0$

[8] J.F. Gomez-Aguilar, J.J. Rosales-Garcia, J.J. Bernal-Alvarado, T. Cordova-Fraga, R. Guzman-Cabrera, Rev. Mex. Fis., 58, 348 (2012).
[9] J.E. Escalante-Martinez, J.F. Gomez-Aguilar, C. CalderonRamon, L.J. Morales-Mendoza, I. Cruz-Orduna, J.R. LagunaCamacho, Adv. Mech. Eng., 8 (4), 1 (2016). DOI: $10.1177 / 1687814016643068$

[10] M. Berman, L.S. Cederbaum, Physica A, 505, 744 (2018). DOI: $10.1016 /$ j.physa.2018.03.044

[11] S. Rekhviashvili, A. Pskhu, P. Agarwal, Sh. Jain, Turk. J. Phys., 43 (3), 236 (2019). DOI: 10.3906/fiz-1811-16

[12] А.В. Псху, С.Ш. Рехвиашвили, Письма в ЖТФ, 45 (1), 34 (2019). DOI: 10.21883/PJTF.2019.01.47154.17540 [A.V. Pskhu, S.Sh. Rekhviashvili, Tech. Phys. Lett., 44 (12), 1218 (2018). DOI: 10.1134/S1063785019010164].

[13] С.Ш. Рехвиашвили, А.В. Псху, ЖТФ, 89 (9), 1314 (2019). DOI: $\quad 10.21883 / J T F .2019 .09 .48055 .284-18 \quad$ [S.Sh. Rekhviashvili, A.V. Pskhu, Tech. Phys., 64 (9), 1237 (2019). DOI: $10.1134 / \mathrm{S} 1063784219090135$

[14] Р.И. Паровик, Письма в ЖТФ, 45 (13), 25 (2019). DOI: 10.21883/PJTF.2019.13.47953.17811 [R.I. Parovik, Tech. Phys. Lett., 45 (7), 660 (2019). DOI: $10.1134 / \mathrm{S} 1063785019070095]$.

[15] Р.И. Паровик, ЖТФ, 90 (7), 1059 (2020). DOI: $\quad 10.21883 /$ JTF.2020.07.49436.233-19 [R.I. Parovik, Tech. Phys., 65 (7), 1015 (2020). DOI: 10.1134/S1063784220070154].

[16] R. Parovik, Mathematics, 8 (11), $1879 \quad$ (2020). DOI: $10.3390 /$ math 8111879

[17] L.F.A. da Silva, V.R. Pedrozo, Jr., J.V.B. Ferreira, Chin. J. Phys., 66, 673 (2020). DOI: 10.1016/j.cjph.2020.04.020

[18] J. Jackson, Classical electrodynamics (Wiley, N.Y., 1999).

[19] Б.И. Степанов, В.П. Грибковский, Введение в теорию люминесцениии (Изд-во АН БССР, Минск, 1963).

[20] Р.Р. Нигматуллин, ТМФ, 90 (3), 354 (1992). DOI: $10.1007 / \mathrm{BF} 01036529$

[21] А.И. Олемской, А.Я. Флат, УФН, 163 (12), 1 (1993). DOI: 10.1070/PU1993v036n12ABEH002208

[22] С.Г. Самко, А.А. Килбас, О.И. Маричев, Интегралы $и$ производные дробного порядка и некоторые их приложсения (Наука и техника, Минск, 1987).

[23] А.М. Нахушев, Дробное исчисление и его применение (Физматлит, М., 2003).

[24] А.В. Псху, Уравнения в частных производных дробного порядка (Наука, М., 2005).

[25] В.В. Учайкин, Метод дробных производных (Артишок, Ульяновск, 2008).

[26] В.Е. Тарасов, Модели теоретической физики с интегро-дифберенцированием дробного порядка (РХД, М.-Ижевск, 2010).

[27] А.Ю. Попов, А.М. Седлецкий, Современная математика. Фундаментальные направления, 40, 3 (2011). DOI: $10.1007 / \mathrm{s} 10958-013-1255-3$ 